\title{
Some Results on Prime and Distinct Prime Distance Labeling of Graphs
}

\author{
Parthiban A., Samdanielthompson G., Ajaz Ahmad Pir, IhsanUlHaq
}

\begin{abstract}
Let $Z$ be the set of all integers. A graph $H$ is a prime distance graph if there exists an injective function $\mathrm{g}: \mathrm{V}(\mathrm{H}) \rightarrow Z$ such that for any two adjacent vertices $x$ and $y$, the integer $|g(x)-g(y)|$ is a prime. So $H$ is a prime distance graph if and only if there exists a prime distance labeling of $H$. If the edge labels of $H$ are also distinct, then $g$ is called a distinct prime distance labeling of $\mathrm{H}$ and $\mathrm{H}$ is called a distinct prime distance graph. The generalized Petersen graphs $P(n, k)$ are defined to be a graph on $2 n(n \geq 3)$ vertices with $V(P(n, k))=\left\{v_{i}, u_{i}: 0 \leq\right.$ $i \leq n-1\}$ and $E(P(n, k))=\left\{v_{i} v_{i+1}, v_{i} u_{i}, u_{i} u_{i+k}: 0 \leq i \leq n-\right.$ 1 , subscripts modulo $n\}$. In this paper, we show that the generalized Petersen graphs $P(n, 3)$ permit a prime distance labeling for all even $n>5$ and conjecture that $P(n, 2)$ and $P(n, 3)$ admit a prime distance labeling for any $n \geq 5$ and all odd $n \geq 5$, respectively. We also prove that the cycle $C_{n}$ admits $a$ distinct prime distance labeling for all $n \geq 3$, besides establishing the prime distance labeling for some graphs.
\end{abstract}

Keywords: Prime Distance Graphs, Prime Distance Labeling, Distinct Prime Distance Labeling, Generalized Petersen Graphs.

\section{INTRODUCTION}

On ly simple, finite, connected, and undirected graphs are considered throughout this paper. The concept of distance graph was introduced by Eggleton et al. [1]. Let $D$ be a subset of the set of all positive integers. The integer distance graph $G(Z, D)$ is the graph with vertex set $Z$ (the set of all integers) and two vertices $s$ and $t$ are adjacent if and only if $|s-t| \in$ $D$. Then the prime distance graph $G(Z, P)$ is the distance graph with $D=P$, the set of all primes. For a detailed study on integer distance and prime distance graphs, see [13-15].

Laison et al. [4] considered the finite subgraphs of $G(Z, P)$. They defined that a graph $H$ is a prime distance graph if there exists an injective function $g: V(H) \rightarrow Z$ such that for any two vertices $s$ and $t$ which are adjacent, the integer $|g(s)-g(t)|$ is a prime number and $g$ is called a prime distance labeling of $H$. So $H$ is a prime distance graph if and only if there exists a prime distance labeling of $H$. Note that in a prime distance labeling, the labels on the vertices of $H$ must be distinct, but the edge labels need not be so. For more results on prime distance labeling one can see [6-10].

Revised Manuscript Received on December 15, 2019

* Correspondence Author

Parthiban A.*, Department of Mathematics,Lovely Professional University,Phagwara-144 411, Punjab, India.Email:mathparthi@gmail.com

Samdanielthompson G., Department of Mathematics,Hindustan College of Arts and Science, Padur, Kelambakkam, Chennai-603 103, India.

Ajaz Ahmad Pir, Department of Mathematics,Lovely Professional University,Phagwara-144 411, Punjab, India.Email: pirajazahmad789@gmail.com

IhsanUlHaq, Department of Mathematics,Lovely Professional University,Phagwara-144 411, Punjab, India.Email: haqihsan95@gmail.com Email:.samdanielthompson@gmail.com

The generalized Petersen graphs $P(n, k)$ are defined to be a graph on $2 n(n \geq 3)$ vertices with $V(P(n, k))=\left\{v_{i}, u_{i}: 0 \leq\right.$ $i \leq n-1\}$ and $E(P(n, k))=\left\{v_{i} v_{i+1}, v_{i} u_{i}, u_{i} u_{i+k}: 0 \leq i \leq\right.$ $n-1$, subscripts modulo $n\}$ [5]. In this paper we prove that the generalized Petersen graphs $P(n, 3)$ permit a prime distance labeling for all even $n>5$ and conjecture that $P(n, 2)$ and $P(n, 3)$ admit a prime distance labeling for any $n \geq 5$ and all odd $n \geq 5$, respectively. We also establish the prime distance labeling of certain graphs. For the sake of convenience, by GPG, we mean the generalized Petersen graph.

\section{MAIN RESULTS}

First, we recall a few important results concerning prime distance labeling of graphs.

Lemma 1 [4] Everysubgraph of a prime distance graph is also a prime distance graph.

Theorem 1 [4] All bipartite graphs permit a prime distance labelling.

Theorem 2 [7] The wheel graph $W_{n}=C_{n-1} \wedge K_{1}$ does not admit a prime distance labeling for $n \geq 10$.

Theorem 3 [7] The fan graph $F_{(1, n)}$ does not admit a prime distance labeling for $n \geq 11$.

\section{A. Prime Distance Labelling of GPG $P(n, 2)$ and} $\boldsymbol{P}(\boldsymbol{n}, 3)$

Lemma 2 The $\operatorname{GPG} P(n, 2)$ is not bipartite for any $n \geq 5$.

Lemma 3 The $\operatorname{GPG} P(n, 3), n \geq 5$ is bipartite when $n$ is even and not bipartite when $n$ is odd.

Theorem 4 The GPG $P(n, 3)$ permits a prime distance labeling for all even $n>5$.

Proof. The proof is direct from Lemma 3 and Theorem 1.

We propose the following two conjectures which could be proved once one can provide a pattern of prime distance labeling of them. We give the prime distance labeling of $P(7,3)$ and $P(8,2)$ in support of these conjectures (See Figure 1).

Conjecture 1 The $\operatorname{GPG} P(n, 2)$ permits a prime distance labeling for any $n \geq 5$.

Conjecture 2 The $\operatorname{GPG} P(n, 3)$ permits a prime distance labeling for all odd $n \geq 5$.

\section{B. Prime Distance Labelling of Certain Graphs}

Definition 5 [12] The Cartesian product of $G\left(V_{1}, E_{1}\right)$ and $\mathrm{H}\left(\mathrm{V}_{2}, \mathrm{E}_{2}\right), \mathrm{G} \times \mathrm{H}$ is the simple graph with $\mathrm{V}_{1} \times \mathrm{V}_{2}$ as its vertex set and two vertices $(\mathrm{u}, \mathrm{v})$ and $\left(\mathrm{u}^{\prime}, \mathrm{v}^{\prime}\right)$ are joined in $\mathrm{G} \times \mathrm{H}$ if and only if either $\mathrm{u}=\mathrm{u}^{\prime}$ and $\mathrm{v}, \mathrm{v}^{\prime}$ are connected in $\mathrm{H}$, or $\mathrm{u}, \mathrm{u}^{\prime}$ are connected in $\mathrm{G}$ and $\mathrm{v}=\mathrm{v}^{\prime}$.

Definition 6 Let $P_{n}$ be a path. Then the Cartesian product $P_{n} \times P_{m}$, where $m \leq n$, is called a square grid graph. 


\section{Some Results on Prime and Distinct Prime Distance Labeling of Graphs}

Theorem 7 A square grid permits a prime distance labeling for all integer $n \geq 4$.

The proof of Theorem 7 is direct from Theorem 1 .

Definition 8 [12] The triangular grid graph $T_{n}$ is the graph on vertices (i,j, k) with $\mathrm{i}, \mathrm{j}, \mathrm{k}$ being non-negative integers summing to $n$ such that vertices are adjacent if the sum of absolute differences of the coordinates of two vertices is 2 .
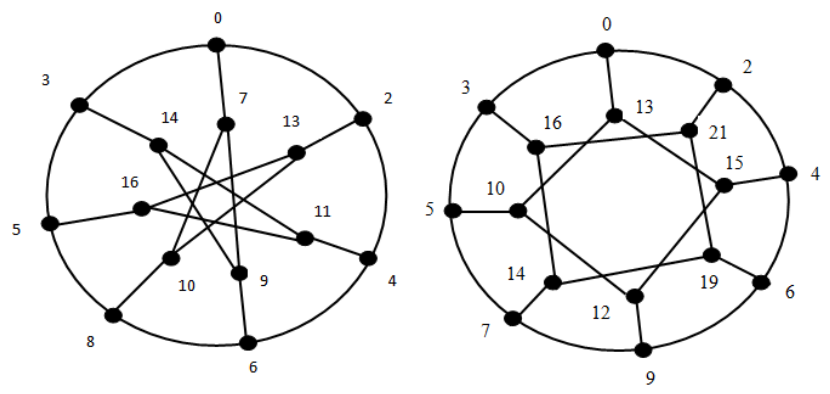

Figure 1A prime distance labeling of the $G P G P(7,3)$ and $P(8,2)$

Theorem 9 A triangular grid $T_{n}$ permits a prime distance labeling for all positive integer $n \geq 1$.

Proof. Let $T_{l}, l \geq 3$ be the given triangular grid. We label the vertices of $T_{l}$ on $l$ levels as $v_{i}^{j}$ for $1 \leq i \leq k$ and $1 \leq j \leq l$. See Figure 2. Define an injective function $f: V\left(T_{l}\right) \rightarrow Z$ as follows: let $f\left(v_{1}^{1}\right)=0$ and $f\left(v_{1}^{j}\right)=f\left(v_{1}^{j-1}\right)+p_{r}, 2 \leq j \leq$ $l, r<l$, where $p_{r}$ are sufficiently large primes (not necessarily distinct). Then $f\left(v_{i}^{j}\right)=f\left(v_{i-1}^{j}\right)+2$ for $2 \leq j \leq l$ and $2 \leq i \leq k$. It is now easy to see that $f$ is the desired prime distance labeling of $T_{l}$.

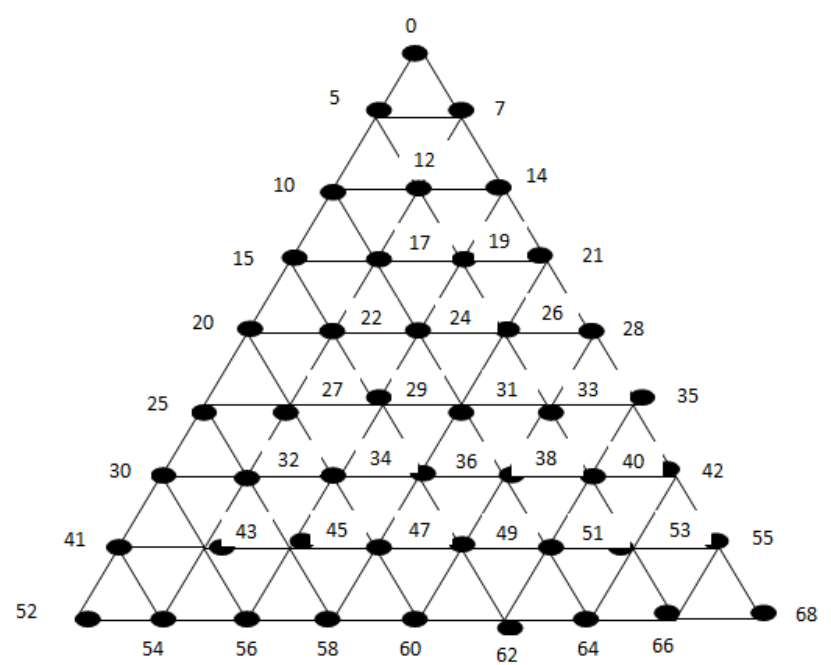

Figure $2 A$ prime distance labeling of the triangular grid $T_{9}$

Definition 10 [3] Let $\mathrm{H}_{1}, \mathrm{H}_{2}, \ldots, \mathrm{H}_{\mathrm{k}}$ be $\mathrm{k}$ copies of a fixed graph $\mathrm{H}$. The grpah $\mathrm{G}$ obtained by adding an edge between $\mathrm{H}_{\mathrm{i}}$ and $\mathrm{H}_{\mathrm{i}+1}$ for $\mathrm{i}=1,2, \ldots, \mathrm{k}-1$ is called the path union of $\mathrm{H}$.

Theorem 11 The graph obtained by the path union of finite number of copies of any prime distance graph admits a prime distance labelling.

Proof. Let $H$ be the prime distance graph on $n_{1}$ vertices with a prime distance labeling $h$. Let $P_{n_{2}}$ be a path on $n_{2}$ vertices. Let $G$ be a graph obtained by the path union of $k(k \geq 2)$ copies of $H$. We label the vertices of the copies of $\mathrm{H}$ as $H_{j}: v_{i}^{j}$ where $1 \leq i \leq n_{1}$ and $1 \leq j \leq k$. Note that $|V(G)|=$ $k|V(H)| \&|E(G)|=k|E(H)|+\mid E\left(P_{n_{2}}\right)$. Define an injective function $f: V(G) \rightarrow Z$ as follows: let $f\left(H_{1}\right)=h\left(H_{1}\right)$. Let $r_{1}=\max \left\{f\left(H_{1}\right)\right\}$ and let $p_{r_{1}}$ be any prime sufficiently larger than $r_{1}$. Next $f\left(H_{2}\right)=f\left(H_{1}\right)+p_{r_{1}}$. Continuing the same process, let $r_{k-1}=\max \left\{f\left(H_{k-1}\right)\right\}$ and let $p_{r_{k-1}}$ be any prime sufficiently larger than $r_{k-1}$. Next $f\left(H_{k}\right)=$ $f\left(H_{k-1}\right)+p_{r_{k-1}}$. Thus $f$ is the desired prime distance labeling of $G$.

Definition 12 [2] A Mongolian tent is a graph formed from $\mathrm{P}_{\mathrm{m}} \times \mathrm{P}_{\mathrm{n}}$ by introducing one extra vertex above the grid and joining every other vertex of the top row of $\mathrm{P}_{\mathrm{m}} \times \mathrm{P}_{\mathrm{n}}$ to the new vertex.

Lemma 4 Let $M(m, n)$ be the mangolian tent. Then $M(2, n)$ permits a prime distance labeling for $1 \leq \mathrm{n} \leq 10$.

Theorem 13 Let $M(m, n)$ be the Mongolian tent. Then $\mathrm{M}(\mathrm{m}, \mathrm{n})$ permits no prime distance labeling for any $\mathrm{m}>0$ and $n \geq 11$.

The proof of Theorem 13 follows easily from Theorem 3 .

Theorem 14 The flower graph $\mathrm{Fl}_{\mathrm{n}}$ admits no prime distance labeling for $\mathrm{n} \geq 5$.

Theorem 15 The sun flower graph $V(n, s, t)$ does not admit a prime distance labeling for $\mathrm{n} \geq 5$.

The proofs of Theorem 14 and Theorem 15 are direct from Theorem 2.

\section{Distinct Prime Distance Labelling of ycles}

In 2017, Parthiban et al. introduced the notion of distinct prime distance labeling of graphs [11]. A graph $H$ is a distinct prime distance graph if there exists an injective function $f_{d}: V(H) \rightarrow Z$ such that for any two vertices $s$ and $t$ which are adjacent in $H$, the integer $\left|f_{d}(s)-f_{d}(t)\right|$ is a prime (distinct). So $H$ is a distinct prime distance graph if and only if there exists a distinct prime distance labeling of $H$ and $f_{d}$ is called a distinct prime distance labeling of $H$. In this section, we prove that the cycles are distinct prime distance graphs. Theorem 16 Let $C_{n}$ be a cycle. Then $C_{n}$ permits a distinct prime distance labeling for all $n \geq 3$.

Proof. Let $C_{n}$ be a cycle on $n \geq 3$ vertices, namely, $v_{1}, v_{2}, \ldots, v_{n}$. We divide the proof in to two cases.

Case 1: When $n \equiv 1(\bmod 2)$

The vertices of the cycles $C_{3}$ and $C_{5}$ can be labeled with the labels $0,2,5$ and $0,2,5,10,17$, respectively in the clockwise direction. So we take $n \geq 7$. Define an injective function $f: V(G) \rightarrow Z$ as follows: without loss of generality, let $f\left(v_{1}\right)=0, f\left(v_{2}\right)=p_{1}, f\left(v_{3}\right)=p_{1}+p_{2}, \ldots, f\left(v_{n-1}\right)=$ $p_{1}+p_{2}+\ldots+p_{n-2}$, and $f\left(v_{n}\right)=p_{1}+p_{2}+\ldots+p_{n-1}$ where $p_{i} \in P$ (the set of all primes) for $1 \leq i \leq n-1$. Then the following two subcases arise.

Subcase 1: If $f\left(v_{n}\right)$ is a prime, then we are through.

Subcase 2: If $f\left(v_{n}\right)$ is not a prime, then add any sufficiently large prime greater than $p_{n-1}$ to $f\left(v_{n-1}\right)$ so as to make the label $f\left(v_{n}\right)$ a prime.

Case 2: When $n \equiv 0(\bmod 2)$

The vertices of the cycles $C_{4}$ and $C_{6}$ can be labeled with the labels $0,7,18,31$ and $0,7,18,31,48,67$, respectively in the clockwise direction. So we take $n \geq 8$. Define an one-to-one function $f: V(G) \rightarrow Z$ as follows: without loss of generality, let $f\left(v_{1}\right)=0, f\left(v_{2}\right)=, f\left(v_{3}\right)=f\left(v_{2}\right)+p_{1}, \ldots, f\left(v_{n-1}\right)=$ $f\left(v_{n-2}\right)+p_{1}+p_{2}+\ldots+p_{n-3}$, and $f\left(v_{n}\right)=f\left(v_{n-1}\right)+$ $p_{1}+p_{2}+\ldots+p_{n-2}$ where $p_{i} \in P$ for $1 \leq i \leq n-2$. Then the following two subcases arise. 
Subcase 1: If $f\left(v_{n}\right)$ is a prime, then the proof is complete. Subcase 2: If $f\left(v_{n}\right)$ is not a prime, then add any sufficiently large prime greater than $p_{n-3}$ to $f\left(v_{n-1}\right)$ so as to make the label $f\left(v_{n}\right)$ a prime. This completes the proof.

\section{III.CONCLUSION}

Prime distance labelling of the generalized Petersen graphs has been investigated, besides establishing the same for other classes of graphs. The distinct prime distance labelling of cycles is also obtained. We believe that the prime distance labelling may find applications in graph-based cryptography.

\section{ACKNOWLEDGMENT}

The authors are very much grateful for the valuable comments of the reviewers.

\section{REFERENCES}

1. R.B. Eggleton, P. Erdos and D.K. Skilton, Colouring Prime Distance Graphs, Graphs and Combinatorics, 6 (1990), pp 17-32.

2. V. Ganesan and K. Balamurugan, Some New Outcomes on Prime Labeling, Int. Research Journal of Eng. and Technology, 3 (11) (2016), pp 819-825.

3. V.J. Kaneria and M. Meghpara, Graceful Labeling for One Point Union for Path of Graphs, International Journal of Mathematics and its Applications, 3(1) (2015), pp 49-54.

4. J.D. Laison, C. Starr, and A. Walker, Finite Prime Distance Graphs and 2-odd Graphs, Discrete Mathematics, 313 (2013), pp 2281-2291.

5. Kh. Md. MominulHaque, Lin Xiaohui, Yang Yuansheng, and Zhao Pingzhong, On the Prime Labeling of Generalized Petersen Graphs $P(n, 3)$, Int. J. Contemp. Math. Sciences, 6 (36) (2011), pp 1783-1800.

6. A. Parthiban and N. Gnanamalar David, On Prime Distance Labeling of Graphs, Lecture Notes in Computer Science, (31) (2017), pp 238-241.

7. A. Parthiban and N. Gnanamalar David, On Finite Prime Distance Graphs, accepted for publication in Indian Journal of Pure and Applied Mathematics, (2017). (To appear)

8. A. Parthiban and N. Gnanamalar David, On Prime Distance Labeling of Some Special Graphs, Contemporary Studies in Discrete Mathematics, 2(1) (2018), pp 21-26.

9. A. Parthiban and N. Gnanamalar David, Prime Distance Labeling of Some Families of Graphs, International Journal of Pure and Applied Mathematics, 118(23) (2018), pp 313-321.

10. A. Parthiban and N. Gnanamalar David, Prime Distance Labeling of Some Path Related Graphs, International Journal of Pure and Applied Mathematics, 120(7) (2018), pp 59-67.

11. A. Parthiban, N. Gnanamalar David, and Atulya Nagar, On Distinct Prime Distance Labeling of Certain Graphs, accepted for publication in the proceedings (AISC series of Springer) of the 9th International Conference on Soft Computing for Problem Solving - SocProS 2019, Liverpool Hope University, Liverpool, UK, September 02-04, 2019.

12. D.B. West, Introduction to Graph Theory, 2nd ed. Englewood Cliffs, NJ: Prentice-Hall, 2000.

13. V. Yegnanarayanan and A. Parthiban, Vertex Coloring of Certain Distance Graphs, International Journal of Pure and Applied Mathematics, 86(4) (2013), pp 669-688.

14. V. Yegnanarayanan and A. Parthiban, Chromatic Number of Graphs with Special Distance Sets-V, Open Journal of Discrete Mathematics, 3 (2013), pp 1-6.

15. V. Yegnanarayanan and A. Parthiban, Chromatic Number of Graphs with Special Distance Sets-III, Journal of Mathematical and Computational Science, 2(5) (2012), pp 1257-1268.

\section{AUTHORS PROFILE}

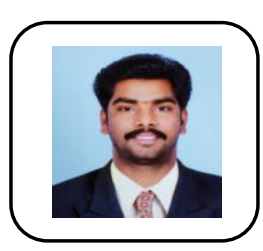

Dr. A. Parthiban born on June 03, 1988. He is presently working as an Assistant Professor in the Department of Mathematics, School of Chemical Engineering and Physical Sciences, Lovely Professional University, Punjab, India He did his Under graduation, Post graduation, and Master of Philosophy at Loyola College, Chennai. He pursued his Doctor of Philosophy (Ph.D) at Madras Christian College, Chennai under the supervision of Prof. N. Gnanamalar David. He has also qualified SET. He is the awardee of UGC-SRF/JRF (RGNF) during 2015-2018.He has worked as a JRF in a NBHM research project at Velammal Engineering College, Chennai during 2011-2013. His area of specialization is graph theory and his research area includes graph labeling, graph coloring, equitable power domination, algebraic graph theory etc. He has more than 25 publications in reputed journals/conference proceedings (SCIE/Scopus/UGC/Peer-reviewed). He has aattended various international and national conferences/workshops across India. He has supervised 2 PG and 2 UG students and presently supervising 4 Ph.D. scholars and 2 PG students.

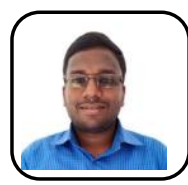

Dr. Samdanielthompson G. born on March 13, 1990 He is Working as Assistant Professor in Department of Mathematics, Hindustan College of Arts, Chennai. He did his Under Graduate, Post Graduate, MPhil and PhD programmes in theDepartment of Mathematics, Madras Christian College, India. He did his PhD under the guide-ship of Prof. N. GnanamalarDavid, Department of Mathematics, Madras Christian College. His research interestsinclude DNA computing, picture languages and graph

languages, graph theory. He has published good number of publication in reputed journals.

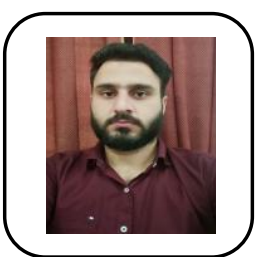

Ajaz Ahmad Pirborn on March 01, 1995. He is the Ph.D. research scholar at the Department of Mathematics, Lovely Professional University, Punjab, India. He did his post graduation at Lovely Professional University, Punjab, India and his under graduation in Govt Degree College, Kupwara, Kashmir. His area of research is graph labeling.

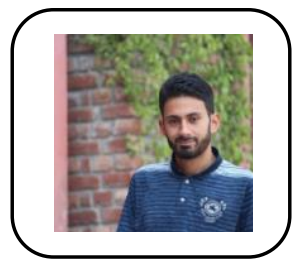

IshanUlHaqborn on March 04, 1996. He is the research scholar at the Department of Mathematics, Lovely Professional University, Punjab, India. He did his under graduation in Govt Degree College, Anantnag, Kashmir and his Post graduation at Lovely Professional University, Punjab, India. His area of research is graph labeling. 